\title{
Dysfunction of Selective Suppression of Auto-Antibody Production in SLE Mouse and Reconstruction of this Mechanism by Induction of Bone- Marrow Chimerism
}

\section{Emiko Takeuchi ${ }^{1 *}$ and Yasuo Takeuchi ${ }^{2}$}

${ }^{1}$ Department of Immunology, Kitasato University School of Medicine, Sagamihara, Kanagawa, Japan

${ }^{2}$ Department of Nephrology, Kitasato University School of Medicine, Sagamihara, Kanagawa, Japan

\begin{abstract}
Background: Mixed chimerism induced by hematopoietic stem cell transplantation is useful in the treatment of autoimmune diseases, but the details of the mechanism by which mixed chimerism reverses the autoimmune state have not been determined. Here, we propose a potential mechanism in which newly developed T cells positively selected by the recipient's thymus are able to regulate auto-reactive $B$ cells. To test this hypothesis, we investigated whether major histocompatibility complex (MHC)/T cell receptor (TCR) cognate interaction was important for the regulation of auto-reactive $B$ cells. We induced an auto-cross-reactive Antibody (Ab) by immunization using a foreign antigen with a homolog to an auto-antigen in a BXSB lupus mouse strain and achieved MHC-matched or mismatched mixed chimerism.
\end{abstract}

Methods: Stable multi-lineage mixed chimeric BXSB mice were established with non-lymphoablative conditioning. In order to induce auto-cross-reactive $A b$, a foreign antigen Pigeon Cytochrome $C$ (PCC) was immunized in these chimeric BXSB mice with various MHC combinations. Anti-PCC and anti-Mouse Cytochrome $\mathrm{C}$ (MCC: cross-reactive with PCC) Abs were measured by ELISA.

Results: In normal mice and MHC-matched/haplo-identical chimeric mice, the titer of the anti-MCC Ab reached plateau at low levels. In BXSB and fully MHC-mismatched chimeric mice, however, both anti-PCC and MCC Ab levels were higher.

Conclusion: These results suggest that the regulatory mechanism selectively suppresses auto-reactive $A b$ production through cognate interaction. Dysfunction of this suppression system is one possible cause of lupus; the induction of BM mixed chimerism may allow us to reconstruct the system.

\section{Introduction}

The etiologic and pathogenic bases of many autoimmune diseases ultimately lie in the self-renewing hematopoietic stem cell population. The effects of bone marrow transplantation (BMT) as a treatment for and/or preventive measure against these autoimmune diseases have been investigated extensively [1]. Studies in animal models show that transfer of hematopoietic stem cells can reverse the autoimmune state but the regimen of hematopoietic transplantation leading to induction of fully allogeneic bone marrow chimerism typically requires stringent conditions that place extreme burdens on the recipient. Moreover, it is known that the fully allogeneic chimeras produced by transplantation of BM cells from a donor fully mismatched at the MHC experience immunodeficiency [2].

Then the induction of allogeneic mixed bone marrow chimerism has been proposed as a treatment for autoimmune disease. Mixed chimerism refers to a state in which allogeneic donor hematopoietic cells coexist with recipient cells. In such animals, specific tolerance to a donor tissue graft and host antigens is induced. Allogeneic mixed chimerism induced by hematopoietic stem cell transplantation not only achieves permanent transplantation tolerance, but also cures autoimmune disease in several animal strains [3-5]. Previously, we reported that the induction of fully MHC-mismatched mixed chimerism could prevent disease development in the BXSB strain, which is known as a mouse model of human systemic lupus erythematosus (SLE) [6,7]. BXSB was one of the first SLE mouse models to be treated by allogeneic BMT. Allogeneic BMT or fully MHC-matched mixed chimerism is known to effectively prevent the development of autoimmune disease in BXSB mice [8-10]. The pathological mechanism through which mixed chimerism effects the suppression of disease development, however, has not been determined. Previously, reporting on one possible candidate mechanism, we indicated that newly developed donor $\mathrm{T}$ cells that are positively selected in host thymus and restricted host MHC could regulate auto-reactive B cells through TCR/MHC cognate interaction [11]. In this report, we investigated whether TCR/MHC cognate interaction plays a significant role in the discrimination between autoreactive and foreign reactive $B$ cells, and in the suppression of autoreactive $B$ cells. We induced an auto-cross-reactive antibody $(A b)$ through the administration of a foreign antigen (pigeon cytochrome C) (PCC) that has homology to an auto-antigen (mouse cytochrome C) (MCC), in BXSB mice and in mixed chimeric BXSB mice with varying MHC combinations. We observed that the auto-cross-reactive $\mathrm{Ab}$ (anti-MCC $\mathrm{Ab}$ ) titer was high only in untreated BXSB mice and fully MHC-mismatched BXSB chimeric mice though the titer did not rise significantly in either fully MHC-matched or haplo-identical chimeric mice. These results suggest that the regulatory mechanism selectively suppressed auto-reactive $\mathrm{Ab}$ production through cognate interaction. Dysfunction in this system is one of the possible causes of lupus in

*Corresponding author: Emiko Takeuchi, Department of Immunology, Kitasato University School of Medicine, 1-15-1 Kitastao, Minamiku, Sagamihara, Kanagawa 252-0374, Japan, Tel: +81 (0)427-78-9266; Fax: +81 (0)427-78-9266; E-mail emichike@med.kitasato-u.ac.jp

Received February 24, 2012; Accepted March 30, 2012; Published April 05, 2012

Citation: Takeuchi E, Takeuchi Y (2012) Dysfunction of Selective Suppression of Auto-Antibody Production in SLE Mouse and Reconstruction of this Mechanism by Induction of Bone-Marrow Chimerism. J Transplant Technol Res S5: 001. doi:10.4172/2161-0991.S5-001

Copyright: ( $) 2012$ Takeuchi E, et al. This is an open-access article distributed under the terms of the Creative Commons Attribution License, which permits unrestricted use, distribution, and reproduction in any medium, provided the original author and source are credited. 
Citation: Takeuchi E, Takeuchi Y (2012) Dysfunction of Selective Suppression of Auto-Antibody Production in SLE Mouse and Reconstruction of this Mechanism by Induction of Bone-Marrow Chimerism. J Transplant Technol Res S5: 001. doi:10.4172/2161-0991.S5-001

Page 2 of 6

BXSB mice, and the induction of bone marrow chimerism may lead to the reconstruction of this suppression system.

\section{Materials and Methods}

\section{Mice}

Male BXSB (H-2 $)$ and B10D2 $\left(\mathrm{H}-2^{\mathrm{d}}\right)$ mice were purchased from Japan SLC, Inc. (Shizuoka, Japan). C3H/HeN $\left(\mathrm{H}-2^{\mathrm{k}}\right)$ and C57BL/6(H$\left.2^{\mathrm{b}}\right)$ mice hereafter referred to as B6 were purchased from CLEA Japan, Inc. (Tokyo, Japan). C57BL/6 (act-EGFP) OsbY01 mice (H-2 $)$ hereafter referred to as GFP/B6 was bred in our animal facility.

\section{Bone-marrow transplantation (BMT)}

Seven-week-old recipient mice were treated with a sublethal dose of $4 \mathrm{~Gy}$ total body X-ray irradiation (TBI) one day before BMT (day-1). Twenty-million bone marrow cells from MHC-mismatched B10D2, MHC-matched GFP/B6 or B10D2 $\times$ GFP/B6 F1 $\left(\mathrm{H}-2^{\mathrm{b} / \mathrm{d}}\right)$ mice were injected intravenously along with a $2.0 \mathrm{mg}$ intraperitoneal (i.p.) administration of hamster anti-mouse CD40L monoclonal antibody (mAb) (MR1, purchased from Bio X Cell, West Lebanon, NH, USA).

\section{Flow cytometric analysis}

Single-cell suspensions of peripheral blood cells from BXSB or transplanted mice were stained with the following fluorochromeconjugated mAbs (BD Biosciences, San Diego, CA, USA) to reveal the indicated surface markers: PE-conjugated B220 (B cells), PE-conjugated anti-CD4 (CD4 ${ }^{+} \mathrm{T}$ cells), anti-CD8 $\left(\mathrm{CD}^{+} \mathrm{T}\right.$ cells), and $\mathrm{PE}$-conjugated anti-CD11b (monocytes/macrophages). Donor derived cells were identified as those that reacted with fluorescein isothiocyanate (FITC)conjugated anti-H-2 $\mathrm{D}^{\mathrm{d}} \mathrm{mAb}$ (34-2-12). Cell suspensions were analyzed with a three-color FACSCalibur flow cytometer. The percentage of chimerism was calculated as the ratio of FITC-positive cells to the total number of applicable PE-positive cells.

\section{Immunization}

Eight weeks after BMT, mixed chimeric BXSB mice with various MHC combinations, and age- matched untreated BXSB mice were immunized with $100 \mu \mathrm{g}$ PCC (Sigma-Aldrich, Tokyo, Japan) emulsified in complete Freund's adjuvant (CFA) (Sigma-Aldrich). These animals were then immunized weekly for seven weeks with PCC alone. The titers of anti-PCC Ab and anti-MCC Ab were measured by ELISA before immunization and at four and eight weeks after the start of immunization. Ten untreated BXSB mice and ten chimeric BXSB mice in each group were examined. In the cases of MHC-matched chimeric mice group and normal control group, more than four mice in each group were examined.

\section{ELISA}

Microtiter plates were coated with $10 \mu \mathrm{g} / \mathrm{ml}$ antigen solution or capture antibody. Purified PCC (Sigma-Aldrich) was used as an antigen to detect anti-PCC Abs. To distinguish auto-reactive anti-MCC Ab from anti-PCC Ab, microtiter plates were coated with Rabbit antimouse cytochrome $\mathrm{C} \mathrm{Ab}$ (Affinity BioReagents, Golden, CO, USA) as a capture Ab. MCC purified from mouse heart was used as an antigen. Wells were washed with PBS containing $0.05 \%$ Tween 20 , blocked with $10 \%$ rabbit serum and then incubated with $50 \mu \mathrm{l}$ of serially diluted serum samples. Washed wells were further incubated with horseradish peroxidase-labeled rabbit anti-mouse Ig. Colorimetric analyses were carried out using tetramethylbenzidine as the substrate. Pooled serum containing $1000 \mathrm{unit} / \mathrm{ml}$ anti-PCC Ab and anti-MCC Ab was used as a standard.

\section{Statistical analyses}

Serum anti-PCC and anti-MCC Ab levels between experimental groups and the untreated group were compared using the nonparametric Mann-Whitney U-test. $\mathrm{P}$ values $<0.05$ were considered to be significant.

\section{Results}

\section{Only auto-cross-reactive antibody production is selectively suppressed}

Cytochrome $\mathrm{C}$, a hemo-protein of the mitochondrial respiratory chain, has been proven to be an extremely useful antigen for immune response studies. Since cytochrome $\mathrm{C}$ is a highly conserved protein, most epitopes of this antigen are commonly found in different species. Pigeon cytochrome C (PCC) also has homology to mouse cytochrome C (MCC) with only six of the 105 amino acids constructing PCC differing from those constructing MCC. However, immunization with PCC can induce anti-PCC Ab production as an immune response to a foreign antigen in several strains of mice. A mouse strain that has a $\mathrm{H}-2^{\mathrm{k}}$ back-ground is known to be a high responder to PCC [12]. To investigate whether anti-PCC $\mathrm{Ab}$ produced by $\mathrm{H}-2^{\mathrm{k}}$ mice in response to immunization with PCC contained an Ab cross-reactive with MCC, $\mathrm{C} 3 \mathrm{H} / \mathrm{HeN}\left(\mathrm{H}-2^{\mathrm{k}}\right)$ mice were immunized with PCC emulsified in CFA, and were then immunized weekly for four weeks with PCC alone. As shown in Figure 1, the anti-PCC Ab induced foreign antigen PCC containing an auto-cross-reactive anti-MCC $\mathrm{Ab}$. When $\mathrm{C} 3 \mathrm{H} / \mathrm{HeN}$ mice were immunized with MCC, however, anti-MCC Ab production was not induced, indicating that MCC itself was not immunogenic for $\mathrm{C} 3 \mathrm{H} / \mathrm{HeN}$ mice (data not shown). On the other hand, immunization with PCC did stimulate helper T cells specific for a non-self epitope of $\mathrm{PCC}$, and both anti-non-self $\mathrm{Ab}$ and auto-cross-reactive $\mathrm{Ab}$ production were induced. These results reveal the existence of functional anti-self $B$ cell clones in the normal immune system. However, as shown in Figure 1, the titer of MCC-reactive Ab reached a low-level plateau and did not rise, while the non-cross-reactive anti-PCC Ab rose to much higher levels. This result suggests the existence of a certain regulatory mechanism that selectively suppresses auto-reactive Ab production.

\section{Dysfunction of selective suppression of auto-antibody production in SLE mouse}

On the other hand, mouse strains with $\mathrm{H}-2^{\mathrm{b}}, \mathrm{H}-2^{\mathrm{d}}$, and these $\mathrm{F} 1$ $\left(\mathrm{H}-2^{\mathrm{b} / \mathrm{d}}\right)$ back grounds are known to be low or non-responders to PCC [12]. B6 is a genetically normal $\mathrm{H}-2^{\mathrm{b}}$ mouse strain, and B10D2 has the same genetic background as B6 except for the $\mathrm{H}-2^{\mathrm{d}} \mathrm{MHC}$. These mice were first immunized with PCC emulsified in CFA and then immunized weekly for seven weeks with PCC alone. The serum antiPCC and anti-MCC Ab levels were measured by ELISA. As shown in Figure $2 \mathrm{~A}$, anti-PCC $\mathrm{Ab}$ production was not induced in $\mathrm{B} 6$ mice. (The same results were observed in $\mathrm{B} 10 \mathrm{D} 2$ and $\mathrm{B} 6 \times \mathrm{B} 10 \mathrm{D} 2 \mathrm{~F} 1\left(\mathrm{H}-2^{\mathrm{b} / \mathrm{d}}\right)$ mice (data not shown). It is thought that since helper T cells which have an $\mathrm{H}-2^{\mathrm{b}}$ or $\mathrm{H}-2^{\mathrm{d}}$ background cannot recognize immunogenic epitopes of PCC as well as T cells with $\mathrm{H}-2^{\mathrm{k}}$ MHC can, immunization with PCC did not induce anti-PCC Ab production. Although anti-MCC Ab levels were slightly high initially, the production of an anti-MCC Ab was not stimulated despite repeated immunization with PCC, which indicates that these B cell clones were non-functional.

The BXSB strain spontaneously develops a progressive lethal autoimmune disease, similar to human SLE. Since the BXSB lupus mouse has an $\mathrm{H}-2^{\mathrm{b}}$ background, it should be a non-responder to PCC yet when PCC was administered to BXSB mice, anti-PCC Ab levels 
Citation: Takeuchi E, Takeuchi Y (2012) Dysfunction of Selective Suppression of Auto-Antibody Production in SLE Mouse and Reconstruction of this Mechanism by Induction of Bone-Marrow Chimerism. J Transplant Technol Res S5: 001. doi:10.4172/2161-0991.S5-001

Page 3 of 6

rose (Figure 2B). Moreover, anti-MCC Ab levels also rose in half of these mice. These results indicate that the one of the defects in the BXSB lupus mouse may be a dysfunction of the regulatory mechanism that selectively suppresses auto-reactive Ab production.

\section{MHC-matched mixed chimerism, but not MHC-mismatched chimerism, can reconstruct the selective suppression mechanism}

The induction of allogeneic mixed bone marrow chimerism has been proposed as a treatment for autoimmune disease. BXSB was one of the first SLE mouse models to be treated by allogeneic BMT. Allogeneic BMT or fully MHC-matched mixed chimerism is known to effectively prevent the development of autoimmune disease in BXSB mice $[9,10]$. Recently, we reported that administration of MHC-matched or MHCmismatched donor BM to BXSB mice that received $4 \mathrm{~Gy}$ total body irradiation on day- 1 and a single injection of $2.0 \mathrm{mg}$ antiCD $40 \mathrm{~L} \mathrm{mAb}$ (MR1) intraperitoneally on day 0 permitted the induction of permanent mixed chimerism and tolerance [6,11]. As previously reported, eight weeks after BMT, the percentage of donor cells in the periphery was approximately $50 \%$, indicating that allogeneic donor and host B cells had contributed equally to the immune response $[11,13]$.

To investigate whether the induction of mixed chimerism can reverse the autoimmune state, PCC was administered to fully MHCmatched chimeric BXSB mice and to fully MHC-mismatched BXSB mice eight weeks after BMT from GFP/B6 (H-2 $\left.{ }^{\mathrm{b}}\right)$ and B10D2 $\left(\mathrm{H}-2^{\mathrm{d}}\right)$ $\mathrm{BM}$, respectively (Figure $3 \mathrm{~A}$ and $3 \mathrm{~B}$ ). As shown in Figure $3 \mathrm{~A}$, in fully MHC-matched chimeric mice, neither anti-PCC nor anti-MCC Ab levels rose, compared with those of untreated BXSB mice. B10D2 is a mouse strain with the same genetic background as B6, except in terms of MHC molecules. $\mathrm{H}-2^{\mathrm{d}}$ is an MHC haplotype of B10D2, so the latter is a fully MHC-mismatched donor for BXSB. As shown in Figure 3B, in fully MHC-mismatched chimeric mice, the titers of both anti-PCC and anti-MCC $\mathrm{Ab}$ rose to levels that were the same as those of untreated BXSB mice.

Recently, we reported that in fully MHC-mismatched chimeric BXSB mice, the majority of the autoreactive anti-DNA IgM was produced by allogeneic donor B cells, whereas host-type anti-DNA $\mathrm{Ab}$ production was suppressed. The majority of the total serum IgM, on the other hand, was produced by host B cells, suggesting that the production of "normal" serum Ig is dependent on host MHC-restricted T cells [11].

Together, these results indicate that $\mathrm{MHC}$ restriction plays a key role in regulating auto-reactive $\mathrm{Ab}$ production.

\section{BMT from a donor with haplo-identical MHC is as effective as that from a fully MHC-matched donor}

If there is indeed a host-type MHC restriction in the suppression of auto-cross-reactive Ab production, BMT from a donor with partially identical MHC that is sufficient to maintain cognate interaction should be equally effective as BMT from a fully MHC-matched GFP/B6 mouse. To test this hypothesis, BM cells taken from B10D2 $\left(\mathrm{H}-2^{\mathrm{d}}\right) \times \mathrm{GFP} / \mathrm{B} 6$ $\left(\mathrm{H}-2^{\mathrm{b}}\right) \mathrm{F} 1$ mice with haplo-identical $\mathrm{MHC}\left(\mathrm{H}-2^{\mathrm{b} / \mathrm{d}}\right)$ were transplanted to BXSB mice. In this case, all B cells, even those differentiated from donor $\mathrm{BM}$, contained at least one $\mathrm{H}-2^{\mathrm{b}}$ allele.

As shown in Figure 4, neither anti-PCC nor anti-MCC Ab levels rose. These results suggest that the maintenance of TCR/MHC cognate interaction with all $\mathrm{B}$ cells is important in regulating auto-reactive $\mathrm{Ab}$ production, and that reconstruction of the $\mathrm{T}$ cell surveillance system may reverse the autoimmune state of lupus-like disease in the BXSB mouse.

\section{Foreign-reactive antibody is normally produced by host type $B$ cells in mixed bone marrow chimeric mice}

As mentioned above, the fully allogeneic chimeras transplanted from a donor fully mismatched at the MHC level experience immunodeficiency. In fully allogeneic chimerism, all $\mathrm{T}$ cells selected in the host thymus are restricted host-type MHC, so no peripheral T cells interact with donor MHC expressed on donor B cells. However, in mixed allogeneic chimerism, because certain populations of host $\mathrm{B}$ cells remain in periphery, humoral immunity is functionally maintained. To confirm that the production of auto-reactive $A b$ is selectively suppressed but the production of foreign-reactive Ab is not, Sheep RBC (SRBC) were administered intraperitoneally to chimeric BXSB mice. Three days after immunization, serum anti-SRBC Ab was detected by flow cytometry. All combinations of chimeric mice produced Abs that

\section{Anti-PCC and anti-MCC Ab produced by $\mathrm{C} 3 \mathrm{HHeN} /\left(\mathrm{H}_{-} \mathbf{2}^{\mathrm{k}}\right)$} Unit $/ \mathrm{ml}$

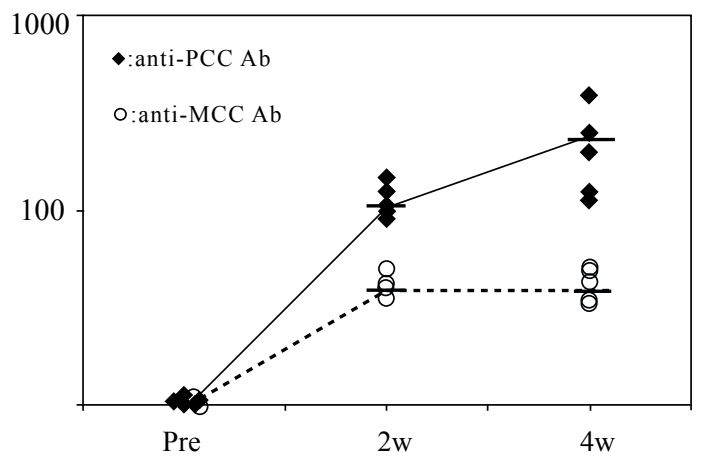

Weeks after immunization

Figure 1: Serum anti-PCC and anti-MCC Ab levels produced by $\mathrm{C} 3 \mathrm{H} / \mathrm{HeN}$ mice $\left(\mathrm{H}-2^{k}\right)$ immunized with $\mathrm{PCC}$. $\mathrm{C} 3 \mathrm{H} / \mathrm{HeN}$ mice $\left(\mathrm{H}-2^{k}\right)$ were immunized with PCC emulsified in CFA, then immunized weekly for four weeks with PCC alone. Anti-PCC and anti-MCC Abs in serum were measured by ELISA before immunization and at two and four weeks after the start of immunization. anti-PCC antibody titers in serum $(n=5)$. $\circ$ : anti-MCC antibody titers in serum $(n=5)$.
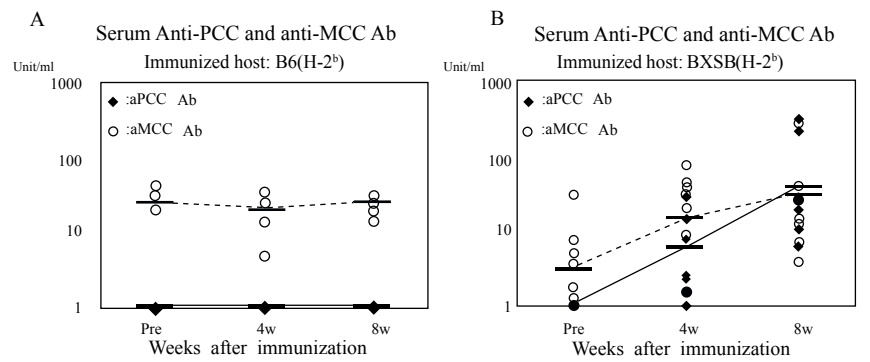

Figure 2: Serum anti-PCC and anti-MCC $A b$ levels produced by $B 6$ and BXSB mice $\left(H-2^{b}\right)$ immunized with PCC. (A) Genetically normal B6 mice $\left(\mathrm{H}-2^{b}\right)$ were immunized with PCC emulsified in CFA, then immunized weekly for seven weeks with PCC alone. Anti-PCC and anti-MCC Ab levels were measured by ELISA before immunization and at four and eight weeks after immunization. : anti-PCC Ab titers in serum. $\triangle$ : anti-MCC Ab titer in serum $(n=4)$. (B) Lupus-prone BXSB mice $\left(H-2^{b}\right)$ were immunized with PCC using the same protocol as for the B6 mice. $\$$ : anti-PCC Ab titers in serum. $\circ$ : antiMCC Ab titers in serum $(n=10)$. 
Citation: Takeuchi E, Takeuchi Y (2012) Dysfunction of Selective Suppression of Auto-Antibody Production in SLE Mouse and Reconstruction of this Mechanism by Induction of Bone-Marrow Chimerism. J Transplant Technol Res S5: 001. doi:10.4172/2161-0991.S5-001

A
Immunized host: Fully MHC-matched chimera
GFP/B6 $\left(\mathrm{H}-2^{b}\right) \rightarrow \mathrm{BXSB}\left(\mathrm{H}-2^{b}\right)$

Serum anti-PCC

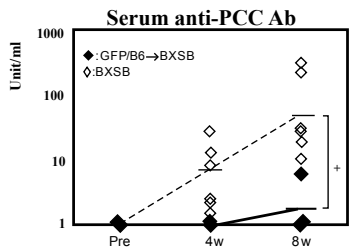

Serum anti-MCC Ab

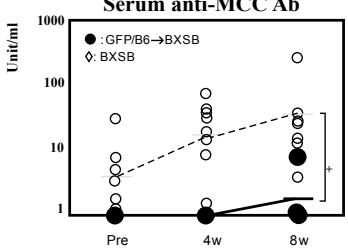

B Immunized host: Fully MHC-mismatched chimera Serum anti-PCC Ab
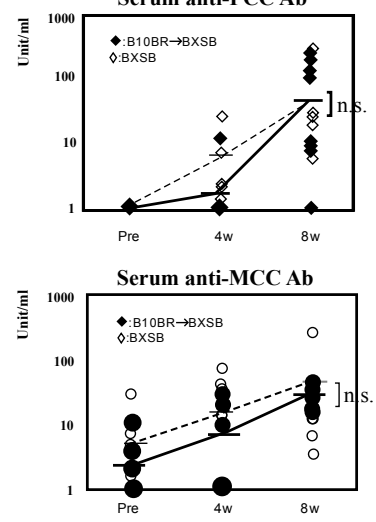
$\operatorname{B10D2}\left(\mathrm{H}-2^{2}\right) \rightarrow \operatorname{BXSB}\left(\mathrm{H}-2^{b}\right)$

Figure 3: Serum anti-PCC and anti-MCC Ab levels produced by fully MHC-matched or fully MHC-mismatched BXSB mice immunized with PCC. BXSB mice were given $4 \mathrm{~Gy}$ TBI on day -1 , followed by twenty-million bone marrow cells (i.v.) together with $2.0 \mathrm{mg}$ anti-CD40L mAb (i.p.). This simple regimen allowed for the induction of stable bone marrow mixed chimerism. Thick bars indicate mean value of $A b$ levels in chimeric mice group. Thin bars indicate mean value of $A b$ levels in untreated BXSB mice group. (A) Upper panel; Fully MHC-matched BXSB chimeric mice that received GFP/B6 bone marrow were immunized with $P C C$ using the same protocol as for the B6 and BXSB mice. Serum anti-PCC and anti-MCC Ab levels between chimeric mice and the untreated BXSB mice were compared. $\checkmark$ : anti-PCC Ab levels in chimeric BXSB mouse serum $(n=5) . \diamond$ : anti-PCC Ab levels in non-treated BXSB mouse serum ( $n-10)$. Lower panel; $\bullet$ : anti-MCC Ab levels in chimeric BXSB mouse serum $(n=5)$. $\circ$ : anti-MCC Ab levels in BXSB mouse serum $(n=10){ }^{*}$ : $p<0.01$ chimeric group vs. non-treated group. (B) Upper panel; Fully MHC-mismatched BXSB chimeric mice that received B10D2 bone marrow were immunized with PCC. Serum anti-PCC and MCC levels were compared with those of untreated BXSB. $\$$ : anti-PCC Ab levels in chimeric BXSB mouse serum $(n=10) . \diamond$ : anti-PCC Ab levels in non-treated BXSB mouse serum $(n=10)$. Lower panel; $\bullet$ : anti-MCC Ab levels in chimeric BXSB mouse serum $(n=10)$. $\circ$ : anti-MCC Ab levels in BXSB mouse serum $(n=10)$. n.s.: no significance.

were reactive with SRBC [11]. As expected, in fully MHC-mismatched mixed chimeras, host-type B cells were reliably activated through immunization with foreign antigen. Therefore, auto-cross-reactive Abs were selectively suppressed but humoral immunodeficiency was not.

\section{Discussion}

BMT is a novel strategy for the control of autoimmune diseases. We and several other groups have shown that the induction of bone marrow mixed chimerism is an effective treatment for and/or means of prevention against the development of autoimmune disease [1,311]. The mechanisms that may be responsible for the reversal of the autoimmune state in BM chimerism have been discussed in previous studies but, the mechanism responsible for the exclusion of self-reactive B lymphocytes has not yet been conclusively identified. To determine the mechanism by which transplanted BM cells prevent the production of auto-reactive Abs, we focused on the role of cognate interactions in the pathogenesis of autoimmune disease in BXSB mice.

In this study, we obtained the following results: 1) Immunization with an allogeneic antigen that has homology to auto-antigen can induce production of auto-cross-reactive Ab in BXSB lupus mice; 2) The induction of mixed BM chimerism with fully MHC-matched or haploidentical donor can selectively suppress the production of autoantibody; and 3) The production of the auto-cross-reactive Ab could not be suppressed in fully MHC-mismatched mixed chimeric mice.
To interpret these results, we propose a T cell surveillance system of mixed chimerism, as illustrated in Figure 5.

$\mathrm{T}$ cell precursors derived from BM differentiate to mature $\mathrm{T}$ cells in the host thymus. Through a process known as positive selection, all $\mathrm{T}$ cell populations can interact with self-MHC with proper affinity. Since these processes are performed by MHC molecules expressed on thymic epithelial cells, the TCR repertoire in mixed chimerism is restricted to the host MHC. We and others speculate that under genetically normal conditions, $\mathrm{T}$ cell-mediated trimming of auto-reactive $\mathrm{Ab}$ production may occur through cognate interactions between TCRs and MHC +
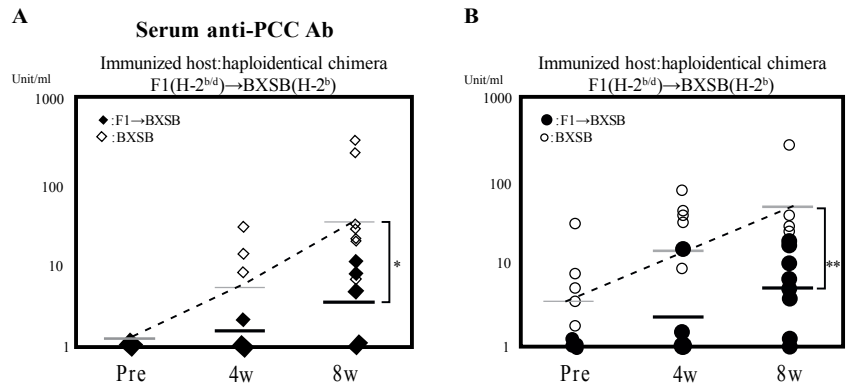

Figure 4: Serum anti-PCC and anti-MCC Ab levels produced by MHC haplo-identical BXSB mice immunized with PCC. Semi-allogeneic BXSB chimeric mice that received GFP/B6 $\times$ B10D2 F1 $\left(\mathrm{H}-2^{\mathrm{b} / \mathrm{d}}\right)$ bone marrow were immunized with PCC. Serum anti-PCC and anti-MCC Ab levels between chimeric mice and the untreated BXSB mice were compared. Thick bars indicate mean value of $A b$ levels in chimeric mice group. Thin bars indicate mean value of $A b$ levels in untreated BXSB mice group. (A) $\downarrow$ : anti-PCC Ab levels in chimeric BXSB mouse serum $(n=10) . \diamond$ : anti-PCC Ab levels in nontreated BXSB mouse serum $(n=10)$. ${ }^{*} p<0.01(B) \bullet$ : anti-MCC Ab levels in chimeric BXSB mouse serum $(n=10)$. $\circ$ : anti-MCC Ab levels in BXSB mouse serum $(n=10){ }^{* *} p<0.05$.

T cell surveilance model of mixed chimerism

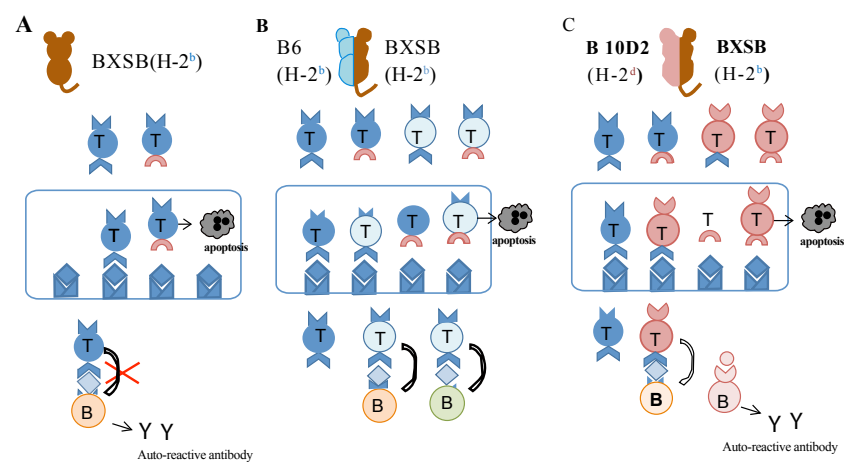

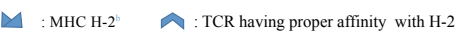

: ${\mathrm{MHC} \mathrm{H}-2^{\triangleleft}}_{2}$ : $\mathrm{TCR}$ having proper affinity with $\mathrm{H}-2$

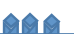

: host thymic epithelial cell

Figure 5: T cell surveillance model of mixed chimerism. (A) In the BXSB lupusprone mouse, mature $T$ cells positively selected in the host thymus can interact with B cells. However, BXSB mice are unable to selectively suppress auto-reactive $\mathrm{Ab}$ production through TCR/MHC interaction. (B) In the fully MHC-matched chimeric BXSB mouse, $T$ cells derived from donor bone marrow can suppress both host and donor auto-reactive $B$ cells through TCR/ $\mathrm{MHC}$ interaction. (C) In the fully MHC-mismatched chimeric BXSB mouse, all peripheral $\mathrm{T}$ cells can recognize host-type $\mathrm{MHC}$ because they were positively selected by host thymic epithelial cells. Therefore, $T$ cells cannot interact with donor $\mathrm{B}$ cells expressing donor-type $\mathrm{MHC}$ or suppress auto-reactive $\mathrm{Ab}$ producing donor B cells. 
Citation: Takeuchi E, Takeuchi Y (2012) Dysfunction of Selective Suppression of Auto-Antibody Production in SLE Mouse and Reconstruction of this Mechanism by Induction of Bone-Marrow Chimerism. J Transplant Technol Res S5: 001. doi:10.4172/2161-0991.S5-001

peptide present on B cells $[14,16]$. In the case of the BXSB mouse, it is known that $\mathrm{T}$ cells, especially $\mathrm{CD} 4^{+} \mathrm{T}$ cells, have certain defects that may play important roles in the pathogenesis of autoimmunity $[17,18]$. We also speculated that the pivotal defect in the BXSB mouse is a genetic defect pertaining to this surveillance function in BXSB T cells (Figure 5A). In the case of fully MHC-matched chimerism, defective surveyor host $\mathrm{T}$ cells can be replaced by $\mathrm{T}$ cells derived from donor BM. Auto-reactive B cells derived from both donor and host BM can be regulated or trimmed by donor T cells through TCR/MHC cognate interactions (Figure 5B).

In the case of $\mathrm{BM}$ mixed chimerism, immature $\mathrm{T}$ cells that have an excessively high affinity for MHC molecules expressed not only on thymic epithelial cells but also on dendritic cells and macrophages, are deleted in a process known as negative selection. In mixed chimeric BXSB mice $\left(\mathrm{H}-2^{\mathrm{b}}\right)$ receiving B10D2 $\left(\mathrm{H}-2^{\mathrm{d}}\right) \mathrm{BM}$, tolerance to both donor and recipient cells can be induced by dendritic cells and macrophages that are developed from both donor and host BM and settled into the thymus. Through negative selection, immature $\mathrm{T}$ cell repertoires, with their strong affinity for the $\mathrm{H}-2^{\text {b }}$ host-type MHC molecule, are deleted by host APCs. Immature $\mathrm{T}$ cells with strong reactivity to the $\mathrm{H}-2^{\mathrm{d}}$ donor-type $\mathrm{MHC}$ molecule, are also deleted by donor APCs, but immature T cells are positively selected on the basis of their weak reactivity with self-peptides presented exclusively on $\mathrm{H}-2^{\mathrm{b}}$ MHC molecules, since thymic epithelial cells express only host MHC. Therefore, in the periphery, all $\mathrm{T}$ cells recognize antigens presented by APCs on $\mathrm{H}-2^{\text {b }}$ MHC molecules, but B cells expressing $\mathrm{H}-2^{\mathrm{d}} \mathrm{MHC}$ are still generated from donor BM because this process is genetically determined. T cells developing in the BXSB thymus should be incapable of cognate interactions with such donor-type $B$ cells with the "wrong" MHC. We speculate that the failure of cognate interaction with $\mathrm{T}$ cells might be the reason why auto-reactive $\mathrm{Ab}$ levels rose in MHC fully mismatched chimeric mice.

This hypothesis, however, does not address the question of how anti-PCC $\mathrm{Ab}$ producing donor $\mathrm{B}$ cells were initially triggered. Other factors derived from the BXSB mouse's own cells that could not be replaced by cells from donor BM seem to accelerate or trigger donor $\mathrm{B}$ cells to produce auto-reactive Abs.

As one possible explanation for this trigger mechanism, we focused on the function of follicular dendritic cells (FDC) of in a BXSB model. FDCs, whose origin is not yet entirely clear, though stomal cells appear to be involved [19], localize to the follicles of secondary lymphoid tissues. FDCs trap antigens in the form of immune complexes (IC) via $\mathrm{Fc}$ and $\mathrm{C}$ receptors. Antigens in FDC-ICs are presented to $\mathrm{B}$ cells and crosslink B cell receptors (BCR) unrelated to MHC molecules $[20,21]$. FDC processing involves protein multimerization rather than cleavage, by arranging surface-bound antigens with a periodicity that is optimal for simultaneously engaging multiple BCRs [22]. As shown in Figure 2, the BXSB mouse spontaneously exhibits a certain level of anti-MCC Ab. Possibly, through immunization of allogeneic antigen PCC, spontaneous anti-MCC Ab/PCC complexes are trapped on FDCs and activated $\mathrm{B}$ cells that have appropriate affinities for either MCC or PCC molecules. In any case, further study is needed to clarify these mechanisms and interactions.

In this paper, we suggested the existence of a $\mathrm{T}$ cell surveillance system that functions through TCR/MHC cognate interaction, through which $\mathrm{T}$ cell-mediated trimming of auto-reactive Ab production can occur under normal conditions. The dysfunction of this system may be a cause of the lupus-like disease in the BXSB mouse. The induction of bone marrow mixed chimerism can reverse the auto-immune state through the reconstruction of this $\mathrm{T}$ cell surveillance system. Furthermore, the maintenance of TCR/MHC interactions with all $\mathrm{B}$ cells is important in regulating auto-reactive $\mathrm{Ab}$ production.

\section{Acknowledgements}

This work was supported in part by a JSPS KAKENHI Grant-in-Aid for Young Scientists (B) 21790950 and by a Kanagawa Nanbyo Fuoundation Grant for young researchers.

\section{References}

1. Sykes M, Nikolic B (2005) Treatment of severe autoimmune disease by stem cell transplantation. Nature 435: 620-627.

2. Onoé K, Fernandes G, Good RA (1980) Humoral and cell-mediated immune responses in fully allogeneic bone marrow chimera in mice. J Exp Med 151 115-132.

3. Mathieu C, Casteels K, Bouillon R, Waer M (1997) Protection agains autoimmune diabetes in mixed bone marrow chimeras. J Immunol 158: 14531457.

4. Sharabi Y, Sachs DH (1989) Mixed chimerism and permanent specific transplantation tolorance induced by a nonlethal preparative regimen. $\mathrm{J}$ Exp Med 169: 493-502.

5. Adachi Y, Inaba M, Amoh Y, Yoshifusa H, Nakamura Y, et al. (1995) Effect of bone marrow transplantation on antiphospholipid antibody syndrome in murine lupus mice. Immunobiology 192: 218-230.

6. Takeuchi E, Takeuchi Y (2007) Allogenic mixed chimerism induced by nonlymphoablative regimen including donor BMT with low-dose TBI and antiCD40L cured proliferative glomerulonephritis in lupus mice. Ann NY Acad Sci 1110: 362-367

7. Takeuchi E (2011) Allogenic mixed chimerism prevented autoimmune thrombocytopenia in BXSB lupus mice receiving donor BMT with nonlymphoablative conditioning of low-dose TBI and anti CD4OL mAb. Kitasato Med J 41: 50-56

8. Wang BY, Cherry, El-Badri NS, Good RA (1997) Prevention of development of autoimmune disease in BXSB mice by mixed bone marrow transplantation. Proc Nat Acad Sci U S A 94: 12065-12069.

9. Wang B, Yamamoto Y, El-Badri NS, Good RA (1999) Effective treatment of autoimmune disease and progressive renal disease by mixed bone-marrow transplantation that establishes a stable mixed chimerism in BXSB recipient mice. Proc Nat Acad Sci U S A 96: 3012-3016.

10. Jones OY, Steele A, Jones JM, Marikar Y, Chang Y, et al. (2004) Nonmyeloablative bone marrow transplantation of BXSB lupus mice using fully matched allogeneic donor cells from green fluorescent protein transgenic mice. $\mathrm{J}$ Immunol 172: 5415-5419.

11. Takeuchi E, Shinohara N, Takeuchi $Y$ (2011) Cognate interaction plays a key role in the surveillance of autoreactive $B$ cells in induced mixed bone marrow chimerism in BXSB lupus mice. Autoimmunity 44: 363-372.

12. Solinger AM, Ultee ME, Margoliash E, Schwartz RH (1979) T-lymphocyte response to cytochrome c. I. Demonstration of a T-cell heteroclitic proliferative response and identification of a topographic antigenic determinant on pigeon cytochrome $\mathrm{c}$ whose immune recognition requires two complementing majo histocompatibility complex-linked immune response genes. J Exp Med 150: 830-848.

13. Takeuchi E (2011) Mixed Hematopoietic Chimerism Allows Cure of Autoimmune Glomerulonephritis: Its Potential and Risks. An Update Glomerulopathies, Rijeka, Croatia, INTECK, Chapter 16: 277-290.

14. Rathmell JC, Townsend SE, Xu JC, Flavell RA, Goodnow CC (1996) Expansion or elimination of B cells in vivo: dual roles for CD40- and Fas (CD95)-Ligands modulated by the B cell antigen receptor. Cell 87: 319-329.

15. Rathmell JC, Cooke MP, Ho WY, Grein J, Townsend SE (1995) CD95 (Fas)dependent elimination of self-reactive $B$ cells upon interaction with CD4+ T cells. Nature 376: 181-184.

16. Shinohara N, Komano H, Lee MH, Tachibana M, Iwata M (1997) CD4+ cells, positive selection, and surveillance of autoantibody production. The Immunologist 5/4: 121-126.

17. Wofsy D (1986) Administration of monoclonal anti-T cell antibodies retards murine lupus in BXSB mice. J Immunol 136: 4554-4560. 
Citation: Takeuchi E, Takeuchi Y (2012) Dysfunction of Selective Suppression of Auto-Antibody Production in SLE Mouse and Reconstruction of this Mechanism by Induction of Bone-Marrow Chimerism. J Transplant Technol Res S5: 001. doi:10.4172/2161-0991.S5-001

18. Smith HR, Chused TM, Smathers PA, Steinberg AD (1983) Evidence for thymic regulation of autoimmunity in BXSB mice: acceleration of disease by neonatal thymectomy. J Immunol 130: 1200-1204.

19. Allen DC, Cyster JG (2008) Follicular dendritic cells networks of primary follicles and germinal centers: phenotype and function. Semin Immunol 20: 14-25.

20. El Shikh ME, El Sayed RM, Szakal AK, Tew JG (2009) T-independent antibody responses to T-dependent antigens: a novel follicular dendritic cell-dependent activity. J Immunol 182: 3482-3491.

21. El Shikh ME, El Sayed RM, Sukumar S, Szakal AK, Tew JG (2010) Activation of $B$ cells by antigens on follicular dendritic cells. Trends Immunol 31: 205-211.

22. Sukumar S, El Shikh ME, Tew JG, Szakal AK (2008) Ultrastructural study of highly enriched follicular dendritic cells reveals their morphology and the periodicity of immune complex binding. Cell Tissue Res 332: 89-99.
This article was originally published in a special issue, Stem/Progenitor Cell Transplantation handled by Editor(s). Dr. R. Goekmen Turan, Rostock University Hospital, Germany 